\title{
DESIGN OF THE BUNCH LENGTH MEASUREMENT FOR THE PHOTO INJECTOR TEST FACILITY AT DESY ZEUTHEN*
}

\author{
Q. Zhao", J. Bähr, I. Bohnet, K. Flöttmann, D. Lipka, D. Richter ${ }^{+}$, F. Stephan, DESY, 22603 \\ Hamburg and 15738 Zeuthen, Germany, ${ }^{+}$BESSY, 12489 Berlin, Germany
}

\begin{abstract}
For measuring the electron bunch length of the photo injector test facility at DESY Zeuthen, a streak camera based system is designed to investigate the longitudinal phase space experimentally at different settings of the photoinjector and make detailed comparisons between theoretical predictions and experimental results. The Cherenkov radiation mechanism is used to convert the electron beam information into a photon beam in the visible range. In order to obtain a better time resolution from Cherenkov radiators, we will employ silica aerogels that have very low refractive index as well as a specially shaped fused silica plate as radiators. The design considerations of the radiators and the optical set-up are discussed with an emphasis on a good time resolution.
\end{abstract}

\section{INTRODUCTION}

The construction and installation of the Photo Injector Test facility at DESY Zeuthen (PITZ) for high-gain shortwavelength free electron lasers and the TESLA linear collider is nearly finished, and it will be commissioned in autumn 2001. PITZ is mainly made up of a 1.5-cell Lband RF gun with emittance compensation techniques and a beam transport and diagnostics system [1,2]. The dedicated Nd:YLF laser system capable of generating micropulses with repetition rate up to $9 \mathrm{MHz}$ illuminates a $\mathrm{Cs}_{2} \mathrm{Te}$ photocathode. This injector will provide an 800 $\mu$ s train of single electron bunches at $1-10 \mathrm{~Hz}$ with a bunch charge of $1 \mathrm{nC}$ and an energy of about 4-6 MeV.

Successful optimization and improvement of the performance of PITZ as well as comparisons between experimental results and theoretical analyses (simulation results) will strongly depend on the ability of beam diagnostics, especially on the measurements of transverse emittance and longitudinal phase space. Therefore, the measurement of micro bunch length is very important for PITZ. Tremendous progress has been made in the measurement techniques, either in time domain or in frequency domain, of micro bunch length in the past decade. Streak cameras are widely used in time domain measurements, which can directly determine the longitudinal bunch charge distribution by detecting the light pulse from the electron bunch through various radiation mechanisms. For the measurement of the micro bunch length at PITZ, the Cherenkov radiation mechanism is used to convert the electron beam information into a photon beam at visible light wavelengths. The produced photon bunches are transported through a $\sim 25 \mathrm{~m}$ long beam line to an optical

\footnotetext{
Supported in part by the HGF-Vernetzungsfonds.

\# email: qiang.zhao@desy.de
}

room and are then detected by either a C5680 or C6138 model of Hamamatsu streak camera. The C5680 can work in either synchroscan or single shot mode with a nominal time resolution of $2 \mathrm{ps}$, while the C6138 has a better nominal time resolution of $200 \mathrm{fs}$ but only in single shot.

\section{CHERENKOV RADIATORS}

Since the energy of the electron beam at PITZ is only about 4-6 MeV, standard optical transition radiation technique produces just a low number of photons which are irradiated in a large solid angle. In order to obtain adequate photon yields after the long photon beamline we will use Cherenkov radiators to convert the information of the electron beam into a photon beam.

\subsection{Photon yield from Cherenkov radiator}

The angular and spectral distribution of the Cherenkov radiation intensity is described by the Tamm formula [3]:

$$
\frac{d^{2} N}{d \Omega \cdot d \lambda}=-\frac{\alpha \cdot n \cdot l^{2}}{\lambda^{3}} \cdot \sin ^{2} \theta \cdot\left(\frac{\sin (k \pi)}{k \pi}\right)^{2}
$$

where:

$-\alpha\left(=\frac{e^{2}}{2 \varepsilon_{0} h c} \approx \frac{1}{137}\right)$ is the fine structure constant;

- $\mathrm{n}$ is the refractive index of the medium;

-1 is the trajectory length of the electron in the medium;

$-\lambda$ is the wavelength of the produced Cherenkov radiation;

- $\theta$ is the angle of observation of radiation with respect to the direction of electron movement;

$-\mathrm{k}=\frac{l}{\beta \cdot \lambda}(1-\beta \cdot n \cdot \cos \theta), \beta$ is the relative velocity of electron;

- $\mathrm{d} \Omega=2 \pi \sin \theta \mathrm{d} \theta$.

Given a $1 \mathrm{~cm}$ thick medium and integrating Eq. 1, we got the relationships between the total number of photons produced from Cherenkov radiation and the refractive index of the medium in different wavelength ranges for a $4 \mathrm{MeV}$ electron beam, as shown in Fig. 1. The full line in Fig. 1 indicates the number of photons with wavelengths between $400 \mathrm{~nm}$ and $650 \mathrm{~nm}$, which is the most sensitive range for the photo cathode in the streak cameras. The shaded lines show the photons from $400 \mathrm{~nm}$ to $410 \mathrm{~nm}$ and from $500 \mathrm{~nm}$ to $510 \mathrm{~nm}$, respectively. Calculations also found the number of photons from a $1 \mathrm{~cm}$ thick Cherenkov radiator with refractive index of 1.03 are over 
4 orders of magnitude higher than that from a metallic transition radiator when the electron beam energy is 4 $\mathrm{MeV}$.

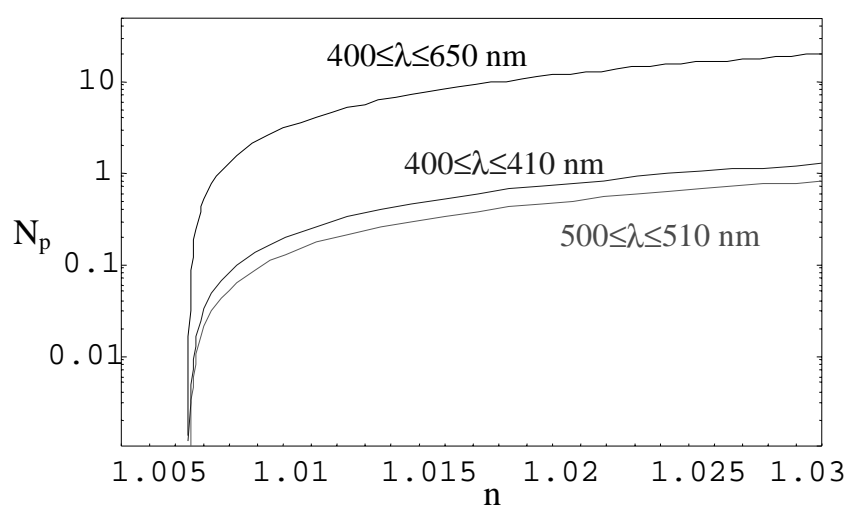

Figure 1. Relationships between the number of photons from Cherenkov radiation and the medium refractive index at different wavelength ranges. A $1 \mathrm{~cm}$ thick Cherenkov radiator hit by a $4 \mathrm{MeV}$ electron beam.

\subsection{Time resolution analysis}

Considering two electrons ( $e_{1}$ and $e_{2}$, see Fig. 2) in a bunch being separated transversely to each other with a distance of $d$ and having the same longitudinal coordinate within the bunch, the time difference $(\Delta t)$ between the Cherenkov light produced by $\mathrm{e}_{1}$ and $\mathrm{e}_{2}$ can be obtained by

$$
\Delta t=\frac{d \cdot n \cdot \sin \theta_{c}}{c}=\frac{d}{c} \cdot \sqrt{n^{2}-\frac{1}{\beta^{2}}}
$$

Where, $\mathrm{c}$ is the velocity of light in vacuum and $\theta_{\mathrm{c}}$ is the Cherenkov angle determined by $\cos \theta_{c}=1 /(\mathrm{n} \cdot \beta)$. To avoid total reflection of the light at exit side of the radiator, the electron beam may have an appropriate incident angle.

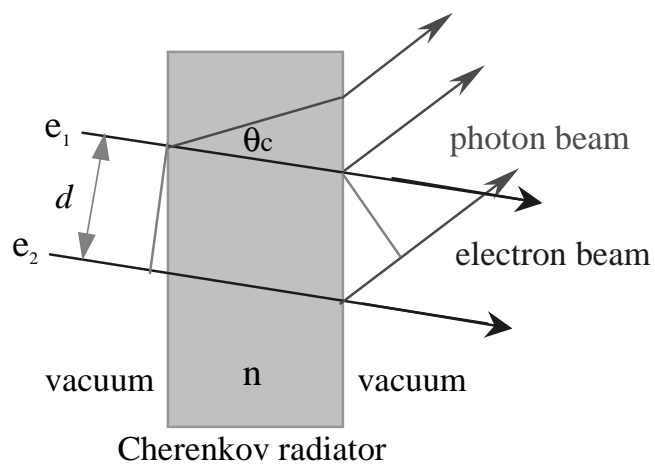

Figure 2. Schematic of the light production by two electrons in a bunch passing through a Cherenkov radiator with refractive index $\mathrm{n}$. $\mathrm{d}$ is the distance between two electrons. $\theta_{\mathrm{c}}$ is the Cherenkov angle.

If a fused silica (refractive index typically around 1.5) is used as radiator, we get $\Delta \mathrm{t}=3.7 \mathrm{ps}$ from Eq. 2. For a 4 $\mathrm{MeV}$ electron beam with beam spot size of $1 \mathrm{~mm}$, the relationship between the refractive index of the radiator (n) and the time dispersion from the radiator $(\Delta t)$ is shown in Fig. 3. In order to achieve a better time resolution from the radiator for the application at PITZ (for example, obtaining the information of the electron bunch resulting from the $1 \mathrm{ps}$ rising and/or falling edges of the laser pulse), we may use a radiator with low refractive index. From Fig. 3, a 200 fs resolution (same as the C6138 streak camera) could be achieved provided the radiator has a refractive index as low as 1.008. Practically only aerogel can be used to reach such low refractive indices.

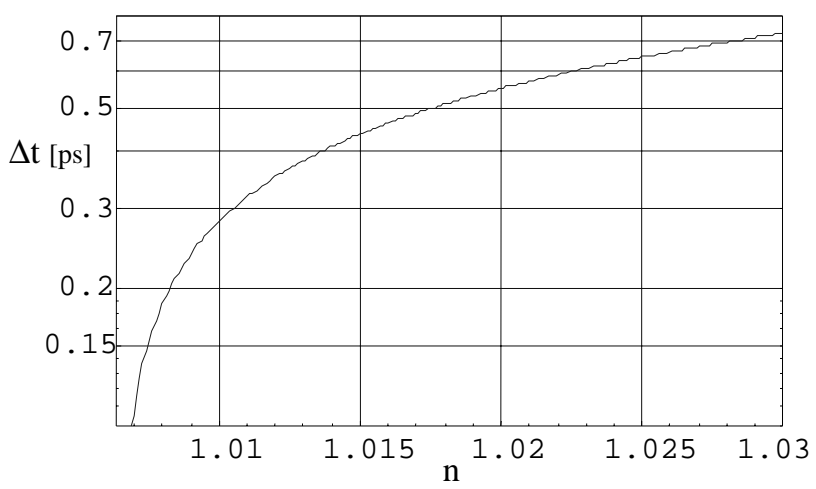

Figure 3. Relationship between the index of refraction (n) and the time dispersion $(\Delta \mathrm{t})$ from a Cherenkov radiator using an electron beam energy of $4 \mathrm{MeV}$ and a beam diameter of $1 \mathrm{~mm}$.

\subsection{Aerogel radiators}

Aerogel, a porous solid material with low refractive index (1.00063 to 1.14), is made up of an aggregation of particles and pores [4]. The size scale of the pores being so small that they scatter very little light and therefore aerogels are highly transparent at visible wavelengths. Because of the specific refractive indices and other unique properties, aerogels have been used in various applications [5].

The refractive indices of silica aerogels for PITZ should be in the range of 1.008 to 1.01 with the tolerance from the mean within \pm 0.0003 and \pm 0.0007 , respectively. The vacuum properties have been tested by measuring the gas load coming from aerogel and estimating its influence

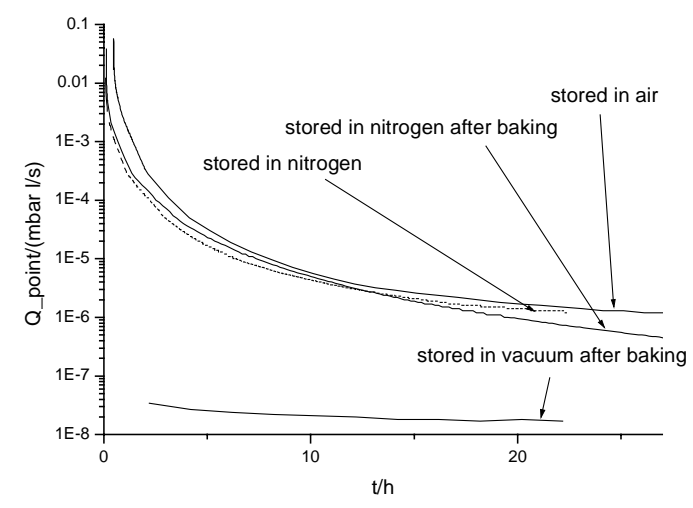

Figure 4. Vacuum test result for silica aerogel: gas load as a function of time for different preparations. 
on the pressure in the cavity section to avoid damage of the $\mathrm{Cs}_{2}$ Te photo cathode. The sample acts generally like a real leak. Only an in-situ bake out results in significant lower desorption rates. During the operation of the injector the temperature of the aerogel will increase caused by electron bombardment. The pressure distribution in the injector was calculated and it shows that either a separate pump or a vacuum tide box around the aerogel might be reqired to maintain good vacuum conditions in the cavity section. Although calculations on the heat dissipation of the aerogels for PITZ indicated silica aerogel radiators could endure the thermal energy from the electron beam, a real beam test has been arranged.

\subsection{Fused silica radiator}

Since the time dispersion of the Cherenkov light results from a correlated wave front distortion, if it is properly compensated, one could also obtain a good time resolution. Fig. 5 illustrates another solution that employs a fused silica radiator with a specially designed surface on the electron-exit side to make up for the time dispersion. Given the desired surface is appropriately machined, the resolution can also be as low as $200 \mathrm{fs}$.

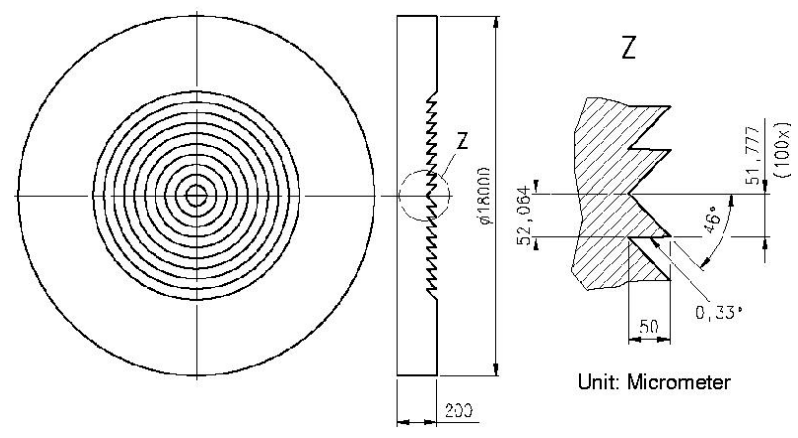

Figure 5. Schematic of the specially shaped fused silica plate serving as Cherenkov radiator

\section{OPTICAL SET-UP}

For analysing charge distributions of electron bunches, the electrons pass through a Cherenkov radiator and emit photons. The produced visible light has to be transmitted from the tunnel through a $\sim 25 \mathrm{~m}$ long beam line to the optical room where it will be measured by a streak camera.

The density of yielded photons at PITZ is not so high and the light is not a point source but a cone with Cherenkov angle. Furthermore, the Cherenkov light covers broadband wavelengths, even in the visible range, the optical elements and air in the long transport line may normally bring about chromatic effect and other aberrations. Therefore, the transport system should have a high light collection and transmission efficiency. The beam line should also be designed to have a high degree of optical correction and low transit time spread due to the difference of the light path. The dispersion within the optical wavelengths should be small $(<<1 \mathrm{ps})$. It is the idea to combine the whole system out of highly corrected subsystems near to diffraction limit. This should lead to a moderate optical resolution of the whole system, which will be remarkable higher as for a design consisting only of single lenses or achromats. The first lens should have a high aperture to collect a maximum of light, whereby the focus length has to match to the port geometry. This light should not be lost by absorption and vignetting in the subsequent elements. Therefore, the number of optical elements should be minimized. Finally, the output focal length and aperture should match the slit of the streak camera and its input optical system. The light is projected in a focus on the slit to maximize the number of photons penetrating the small slit. This light concentration in the focus is limited by the entrance aperture of the lens in the streak camera, which performs the image of the slit onto the photo cathode. The width of the slit right at the entrance of the streak camera should be chosen under the trade between the requirement of the time resolution and the signal-to-noise ratio. Furthermore, space charge effects in the streak tube have to be avoided by appropriate setting of the optical system.

For the high temporal resolution, narrow width of bandpass filters are usually needed in the measurement in order to avoid the pulse broadening due to optical dispersion in the lens and air.

The trigger driving the streak camera will be generated from the master oscillator. The time jitter between the RF (laser) and the camera should be as small as possible. Therefore, the phase stability of the gun is also very important for the bunch length measurement.

\section{SUMMARY}

Cherenkov radiators with both silica aerogels and fused silica plate are designed for the measurement of electron bunch lengths with high time resolution. The long light transport line is optimized to have a high collection efficiency, high transmission, low aberration and low dispersion. In order to measure the longitudinal phase space, the streak camera will detect photon beams from a Cherenkov radiator after an analysis magnet so that both the distributions of electron momentum and longitudinal profile can be measured simultaneously.

\section{ACKNOWLEDGEMENTS}

The authors would like to thank Drs. G. Poelz, R. Kaiser and A. Donat of DESY for their help and advice on aerogels. One of the authors (QZ) appreciates Drs. A. Lumpkin of ANL, L. Hrubesh of LLNL, P. Tsou of NASA and M. Yokoyama of Matsushita Electric Works, Ltd. for the useful discussions and suggestions.

\section{REFERENCES}

[1] F. Stephan, D. Kraemer, I. Will and A. Novokhatski, Proc. FEL'2k, Durham, USA, August 2000.

[2] J. Baehr et al., DIPAC'01, Grenoble, France, May 2001.

[3] I. E. Tamm, J. Phys. USSR 1(1939)439.

[4] http://eande.lbl.gov/ECS/Aerogels

[5] L. Hrubesh, Proc. ISA'98. 